\title{
Histomorphological assessment of non-neoplastic renal diseases at autopsy: an institutional experience in Southwestern Nigeria
}

\author{
Sebastian A. Omenai ${ }^{1}$, Mustapha A. Ajani ${ }^{2,3 *}$, John I. Nwadiokwu ${ }^{2}$, Clement A. Okolo ${ }^{2,3}$ \\ 1. Department of Anatomical Pathology, Edo University, Iyamho, Edo state, Nigeria. \\ 2. Department of Pathology, University College Hospital, Ibadan, Oyo state, Nigeria. \\ 3. Department of Pathology, College of Medicine, University of Ibadan, Ibadan, Oyo state, Nigeria.
}

*Corresponding Author: Mustapha A. Ajani; ajanimustapha42@gmail.com

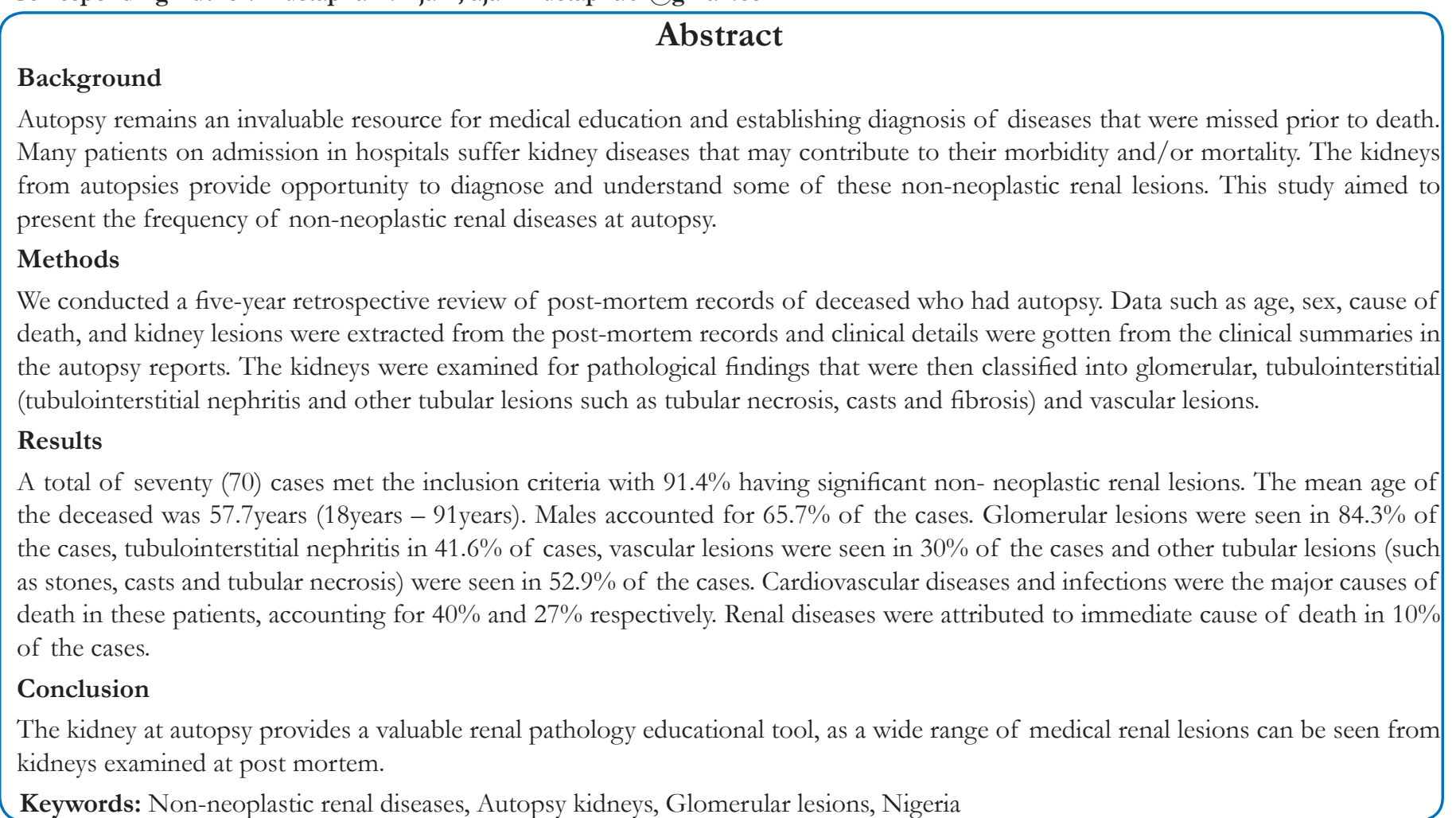

\section{Introduction}

In Nigeria, the prevalence of chronic kidney disease (CKD) from hospital studies is high and ranges from 11.4 to $26 \%{ }^{1}$. Cardiovascular diseases and mortality risks are significantly increased in patients with end stage renal disease (ESRD) and CKD worldwide ${ }^{2}$. Acute kidney injury (AKI) was reported to be seen as high as $82 \%$ in hospitalized paediatric patients and about $12.5 \%$ in hospitalized adult patients ${ }^{3,4}$.

A significant proportion of patients with AKI (58.1\%) presents with stage 3 injuries with the attendant increased risk of mortality and renal morbidity ${ }^{3}$.

Autopsy is still an important diagnostic procedure despite improvements in antemortem diagnosis and modern diagnostic facilities available, missed diagnosis discovered during autopsies is still as high as $44.9 \% 0^{5}$.The subtleness and variability of disease process is the proposed reason for most missed diagnosis as some major pathologies can be present without the classical clinical features ${ }^{5,6}$.

How often do autopsy pathologists report medical renal diseases during autopsies? Or even attribute cause of death to medical renal disease? Henriksen postulated that the examination of kidney and the interpretation by the pathologist is a factor of training in renal pathology and experience ${ }^{7,8}$. Also the primary reason for autopsies in most instances (as there is drop in the rate hospital post -mortems) is to establish the cause of death, such that pathologists pay more attention to the heart, lungs and brain ${ }^{8}$. The findings at post-mortem examination of the kidney might be crucial for the family members of the deceased especially those with genetic components as the kidney is rarely biopsied in many medical renal diseases in critically ill patients ${ }^{7}$.The diseases that are seen in the kidney at autopsy includes glomerular diseases, tubulointerstitial diseases and vascular diseases ${ }^{8}$. Acute tubular necrosis and arterionephrosclerosis are the most commonly noted findings of kidneys at autopsy ${ }^{8,9}$. Divyashree et al., in a study of nephrectomy specimens in India found that non-neoplastic lesions accounted for $72 \%$ of histological diagnoses and that chronic pyelonephritis was the most common non-neoplastic renal lesion in surgical 
nephrectomies ${ }^{10}$

Post-mortem autolysis is a challenge in histopathological assessment of kidneys at autopsies ${ }^{11}$. This post mortem alteration is worse in deceased with high body mass index ${ }^{11}$. This may impact on the assessment of a kidney from autopsies compared to surgical nephrectomy specimens. Despite this, autopsy provides a unique opportunity to confirm clinical suspicion of medical renal disease, establish the effect of systemic disease on the kidney and also diagnose an occult renal pathology $8,12,13$. Most kidney lesions can be identified from gross examination and basic haematoxylin and eosin stains $^{7}$. Electron microscopy, immunofluorescence and even molecular studies will be needed in a minority of cases ${ }^{8,11}$ The spectrum of medical renal diseases that can be congenital, inflammatory or systemic and could be glomerular, vascular or tubulointerstitial or even a combination ${ }^{14-16}$.

Medical renal diseases are frequent during autopsies, although they are mostly underrecognized and significant diagnosis can be missed as well ${ }^{17}$. This study was conducted to describe the histomorphology of non-neoplastic renal diseases at autopsy.

\section{Methods}

This was a five-year retrospective review of post-mortem records of deceased who had autopsy in the Department of Pathology, University College Hospital, Ibadan from January 2015 to December 2019. All cases were clinical autopsies that were conducted after obtaining consent from next of kin of the deceased. Full and complete autopsies involving the kidneys were included in the study. Cases with limited autopsies, inconclusive autopsies and those below 18 years of age were excluded from the study.

Strict confidentiality was maintained in the conduct of this study. Data such as age, sex, cause of death, and kidney lesions were extracted from the post-mortem records and clinical details were gotten from the clinical summaries of the post-mortem reports only. The causes of death were classified into renal, cardiovascular, cerebrovascular, malignancy, infection including sepsis, acute diabetic emergencies, obstetric emergency and accidents. The kidneys were examined and described during the autopsies and routine samples were obtained for histological examinations. The H\&E-stained slides of the kidneys were examined for pathological changes. Findings were then classified into glomerular, tubulointerstitial (tubulointerstitial nephritis and other tubular lesions such as tubular necrosis, casts and fibrosis) and vascular lesions. Special stains including Congo red, mason trichome and PAS were done in some cases as needed.

The data were analysed using the Statistical Package for the Social Sciences (version 23; IBM Corporation, Armonk, New York, USA,2014) and expressed as frequencies, median, means and mode.

\section{Ethical Approval}

This was a retrospective autopsy study. Ethical Approval was not required.

\section{Results}

There was a total of seventy (70) cases that met the inclusion criteria with $91.4 \%$ having significant non neoplastic renal lesions. The mean age of deceased who had post-mortem examination was 57.7 years (18years to 91years). Males accounted for $65.7 \%$ of the cases. Immediate cause of death was attributable to renal pathology in $10 \%$ of the cases with majority of causes of death being cardiovascular (40\%) followed distantly by infectious causes including sepsis at $27.1 \%$. (Figure 1). The renal causes of death were end stage renal disease (ESRD) constituting $5.7 \%$ of all deaths, chronic kidney disease (CKD), obstructive uropathy with CKD, and chronic glomerulonephritis with uraemic encephalopathy accounting for $1.4 \%$ of all deaths respectively.

The most common lesions were glomerular lesions seen in $84.3 \%$ of the cases. Tubular lesions including acute tubular necrosis were reported in $52.9 \%$ of cases, tubulointerstitial nephritis were reported in $41.6 \%$ of the cases, while vascular lesions were reported in $30 \%$ of cases. A single case of congenital renal anomaly $(1.4 \%$ of cases: cystic renal dysplasia; Figure 2) was found in this study. These occurred in variable combination.

The most common glomerular lesion was benign nephrosclerosis accounting for $47.1 \%$ of glomerular pathologies. Chronic glomerulonephritis was the most common primary glomerular disease accounting for $12.9 \%$ of glomerular lesions. (Table 1)

Table 1: Frequency of glomerular lesions in autopsy kidneys

\begin{tabular}{|l|l|l|}
\hline Histological Diagnosis & Frequency & Percentage (\%) \\
\hline Benign nephrosclerosis & 33 & 47.1 \\
\hline Chronic glomerulonephritis & 8 & 12.9 \\
\hline Malignant nephrosclerosis & 7 & 10.0 \\
\hline Diabetic glomerulosclerosis & 5 & 7.1 \\
\hline $\begin{array}{l}\text { Focal segmental } \\
\text { glomerulosclerosis }\end{array}$ & 2 & 2.9 \\
\hline $\begin{array}{l}\text { Me mb ra nop rolife rative } \\
\text { glomerulonephritis }\end{array}$ & 1 & 1.4 \\
\hline $\begin{array}{l}\text { Sickle cell nephropathy } \\
\text { Nil lesion }\end{array}$ & 2 & 2.9 \\
\hline
\end{tabular}

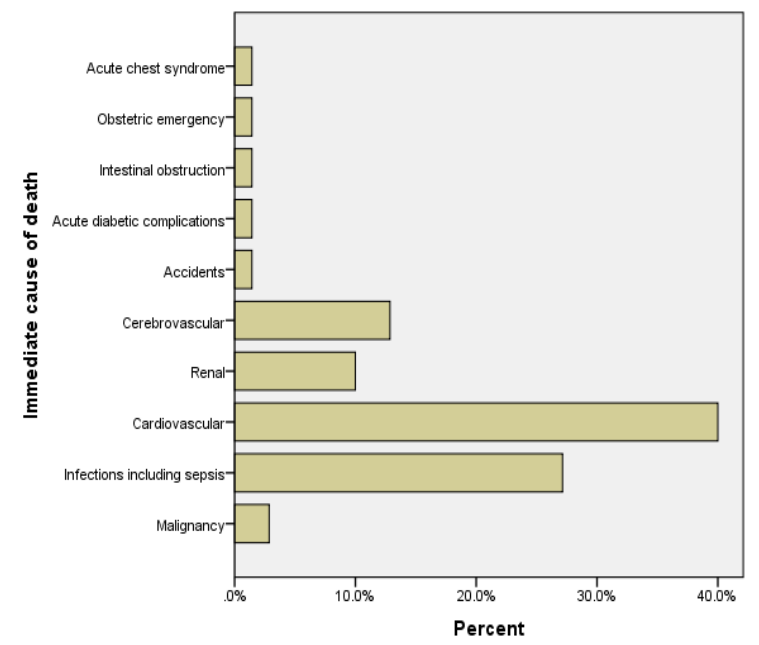

Figure 1. Bar charts showing the immediate causes of death. 
Table 2: Showing frequency of tubular and renal interstitial diseases in $\mathbf{7 0}$ autopsy kidneys

\begin{tabular}{|c|c|c|}
\hline Histological diagnosis & Frequency & Percentage (\%) \\
\hline a. Tubulointerstitial nephritis & & \\
\hline $\begin{array}{l}\text { 1. C hron ic } \\
\text { pyelonephritis }\end{array}$ & 22 & 31.4 \\
\hline 2. $\begin{array}{l}\mathrm{A} C \mathrm{c} \quad \mathrm{u} e \\
\text { pyelonephritis }\end{array}$ & 6 & 8.6 \\
\hline $\begin{array}{l}\text { 3. C h r o n i c } \\
\text { granulomatous } \\
\text { pyelonephritis } \\
\text { 4. Nil pathology }\end{array}$ & 41 & 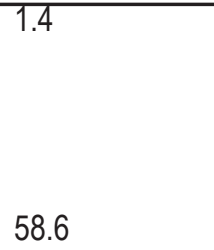 \\
\hline b. Tubular lesions & & \\
\hline $\begin{array}{ll}\text { 1. Acute } \\
\text { necrosis }\end{array}$ tubular & 27 & 38.6 \\
\hline 2. Nephrolithiasis & 2 & 2.9 \\
\hline 3. Hydronephrosis & 2 & 2.9 \\
\hline 4. Simple cortical cyst & 1 & 1.4 \\
\hline $\begin{array}{l}\text { 5. Renal dysplasia } \\
\text { 6. Nil lesion }\end{array}$ & $\begin{array}{l}1 \\
37\end{array}$ & $\begin{array}{l}1.4 \\
52.8\end{array}$ \\
\hline
\end{tabular}

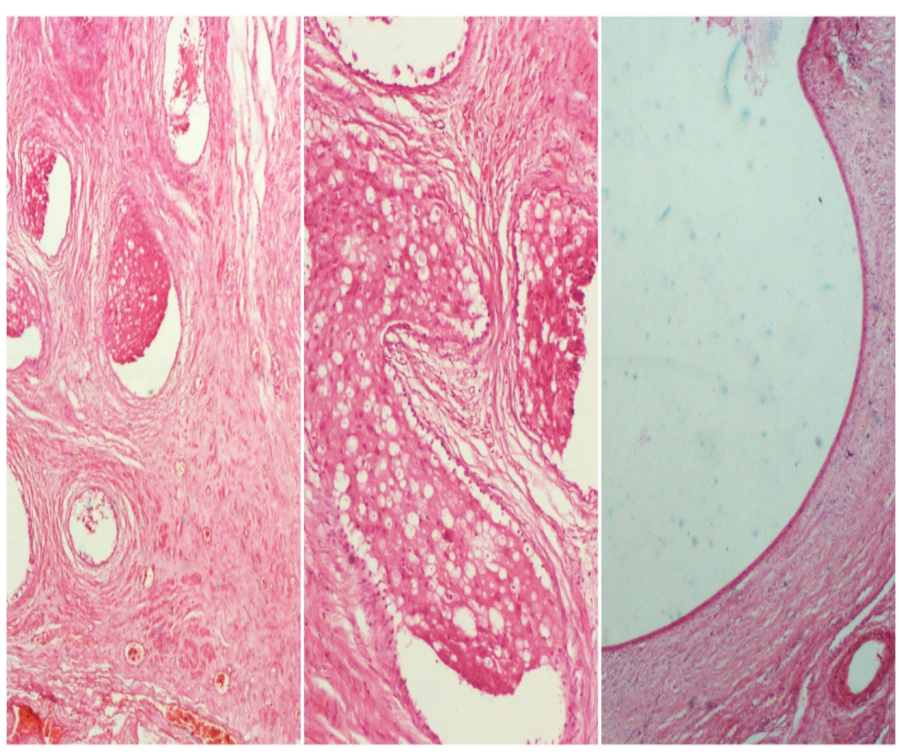

Figure 2: Photomicrograph showing cystic renal dysplasia composed of immature mesenchyme, including cartilage, fibromuscular cuffing of poorly formed ducts and cystically dilated tubules. (Haematoxylin and eosin stains, X100)

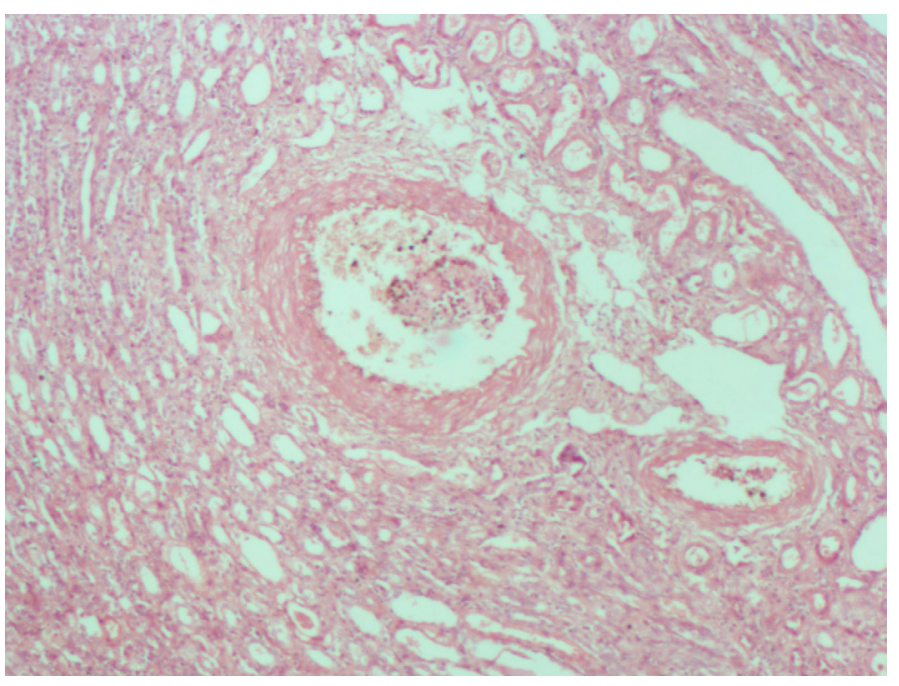

Figure 3: Section of kidney showing hyperplastic arteriolosclerosis. (Haematoxylin and eosin stains, X100)

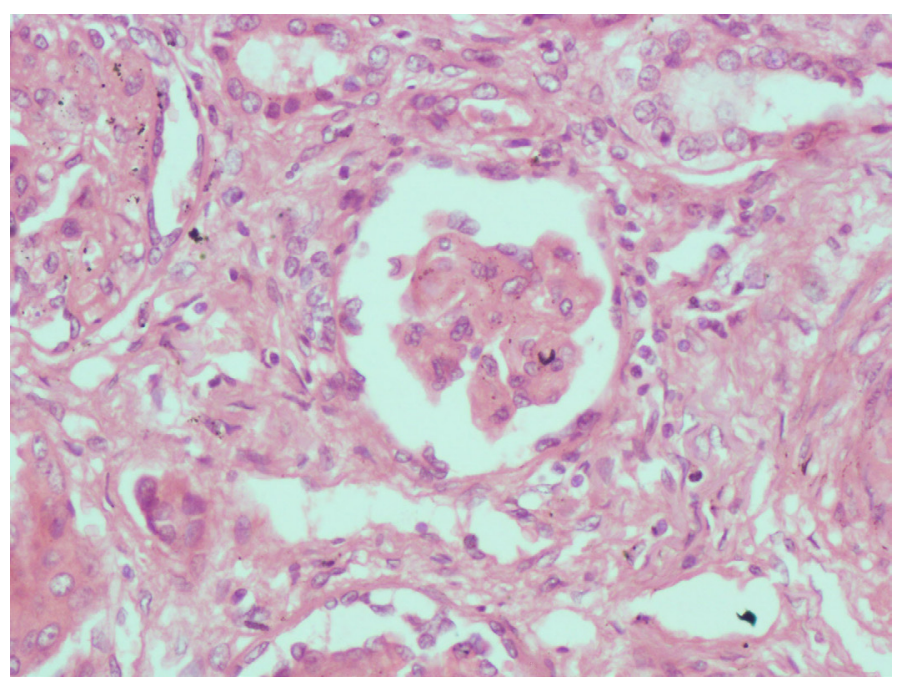

Figure 4: Section of kidney showing diabetic glomerulosclerosis with Kimmelstiel-Wilson nodules (arrows). (Haematoxylin and eosin stains, X100 


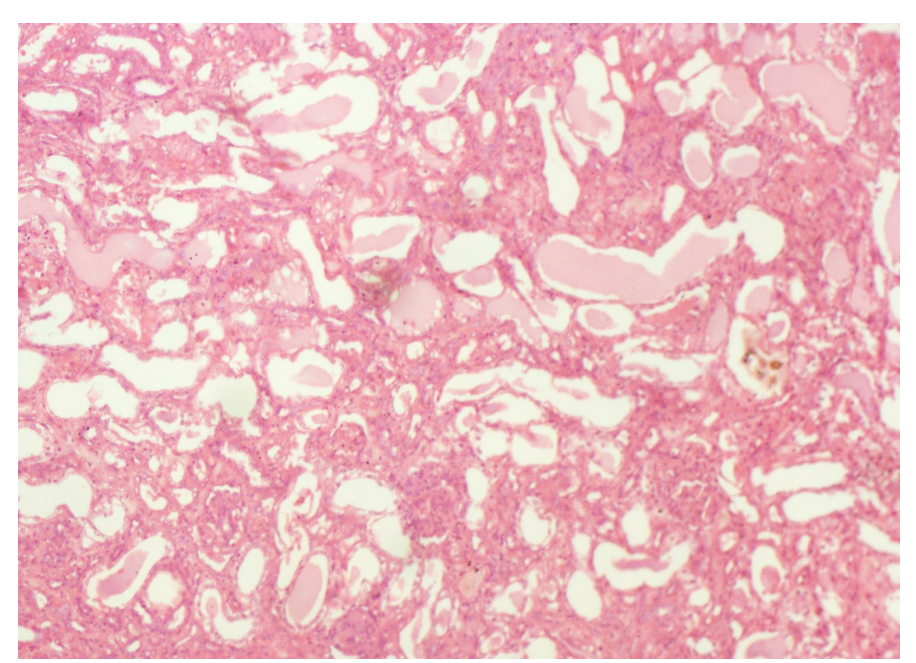

Figure 5: Section of kidney showing thyroidisation of tubules with infiltration of the interstitium by lymphocytes (Chronic pyelonephritis) (Haematoxylin and eosin stains, X100)

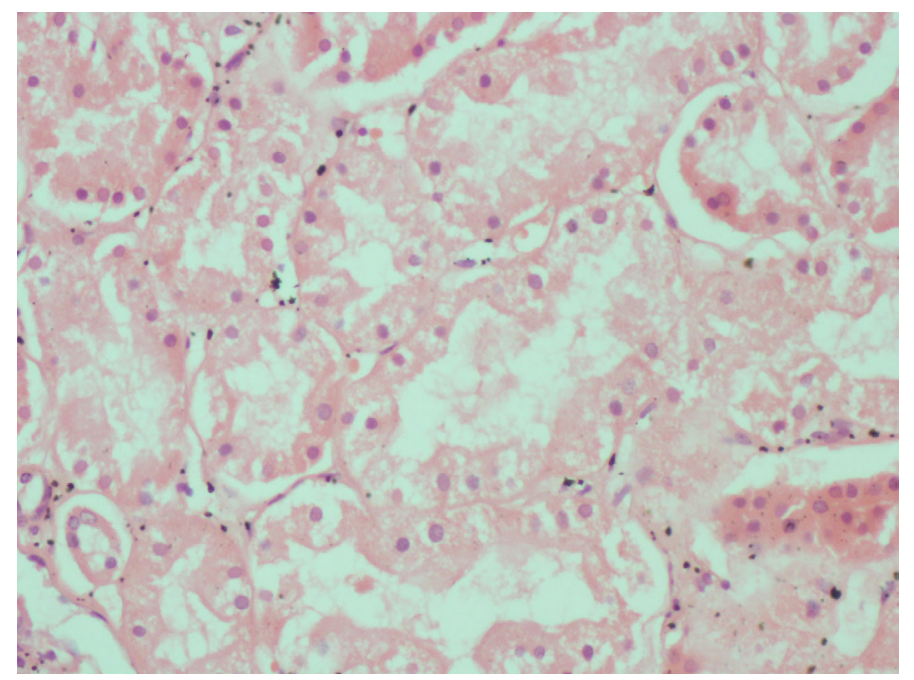

Figure 6: Section of kidney showing acute tubular necrosis. (Haematoxylin and eosin stains, X100)

Figures 3 and 4 show benign nephrosclerosis (Hyperplastic Arteriolosclerosis) and diabetic glomerulosclerosis (Kimmelstiel-Wilson nodules), some of the common glomerular lesions found in this study.

Chronic pyelonephritis with $31.4 \%$ of tubulointerstitial nephritis was the most common inflammatory lesion seen. (Table 2, Figure 5) Among other lesions involving the tubules, acute tubular necrosis '[ATN] (Figure 6) is the most common finding, seen in $38.6 \%$ of the cases. Hyaline arteriosclerosis was the most common reported vascular lesion seen in this review. It was reported in $55.7 \%$ of cases. (Table 3)

\section{Discussion}

Post-mortem examinations are on decline and this is attributable to advances in modern diagnostics ${ }^{8}$. The cases we reviewed in this study show high prevalence of significant medical renal lesions at autopsies. Perrone et al. demonstrated that medical renal diseases are prevalent in autopsied kidneys with a lot of them omitted by the examining pathologist ${ }^{17}$. Significant medical renal diseases were reported in $35 \%$ of their review ${ }^{17}$. The marked difference in prevalence of significant medical renal pathology might be due to the small size of our study sample compared to their study that examined 205 adult autopsies. The importance of noting these changes is the fact that it provides information on the disease process as most critically ill patients are not biopsied for ante-mortem diagnosis usually ${ }^{17}$. It is important that pathologists pay attention to kidney examination as most medical renal lesions are likely to be missed $^{7}$.

Non-neoplastic renal diseases can be as a consequence of systemic disease or primary renal disease ${ }^{13,18,19}$. We had a case of unilateral renal dysplasia in this review (Figure 2), Barakat et al reported a prevalence of $4.6 \%$ for congenital abnormalities seen in autopsies ${ }^{20}$. Kakkar et al., who reviewed paediatric autopsies reported a value of $3.66 \%$ of renal dysplasia in their study ${ }^{14}$. Renal dysplasia is a developmental abnormality containing malformed kidney tissue structures resulting from abnormal interaction between ureteric bud and the mesenchyme ${ }^{19}$. The kidney can be enlarged, normal or reduced in size, this index case was significantly small in size and weighed $45 \mathrm{~kg}$ with compensatory hypertrophy of the contralateral kidney.

Acute tubular necrosis is seen on histology as disruption of the lining epithelium of the tubules ${ }^{11}$ (Figure 6). This is the most common cause of clinical AKI in hospitalized patients and it could be ischaemic or toxic ${ }^{11}$. It results commonly from hypotension, sepsis, endogenous toxins and nephrotoxic drugs such as antibiotics and chemotherapeutic drugs $^{8}$. It is not easy distinguishing autolysis from AKI from autopsy samples. Autolysis commonly is more widespread, show complete detachment of the tubular cells in the lumina with preservation of the brush borders that are easily demonstrated on PAS stain ${ }^{11}$.

Tubulointerstitial nephritis and other tubular lesions was found in approximately $42 \%$ and $53 \%$ of cases respectively. Chronic pyelonephritis is a descriptive term that refers to the presence of chronic inflammation within the tubules and interstitium and scarring due to bacterial infection (Figure $5)^{19}$. It could be obstructive or non-obstructive and it could be specific like granulomatous inflammation or non-specific chronic inflammation. Divyashree et al., in their study reported that chronic non-specific pyelonephritis was the most common tubulointerstitial inflammatory pattern in the kidney accounting for $84 \%$ of the cases, this was very similar to our study that showed that $75 \%$ of tubulointerstitial inflammation were chronic and non-specific ${ }^{10}$. We had a case of chronic granulomatous inflammation as a result of disseminated tuberculosis. This indicates that pathologies affecting the tubules are relatively prevalent which was similar to an autopsy review of patients who had stem cell transplantation ${ }^{13}$. In reviewing kidneys from autopsies while looking out for tubulitis, we should also note and rule out the presence of stones, renal casts and tubular necrosis. They could support the overall disease process that was responsible for the death or could even be the terminal event $^{7}$.

Glomerular lesions were the dominant histological changes seen. Glomerular lesions were present in $84.3 \%$ of the cases which is similar to some other studies ${ }^{6,9,21}$. Truong et al., who examined nephrectomy specimen for non-neoplastic diseases reported that hypertensive nephrosclerosis and diabetic nephropathy were the most frequent lesions ${ }^{22}$. Bonsib et al., showed that non-neoplastic nephropathy were mainly of diabetic origin and nephrosclerosis was the most common lesion seen ${ }^{18}$. In our study benign nephrosclerosis was the predominant glomerular lesion seen and the patients were either hypertensive, diabetic or had both debilitating illnesses. This was similar to the finding of Ueda et al. ${ }^{9}$ Well established diabetic glomerulosclerosis (Figure 4) was seen in about $7 \%$ of all examined kidneys which was similar to 
the $3.7 \%$ reported by Ueda et al. ${ }^{9}$ The reason for this low percentage compared to the study of Bonsib et al., was because in this current study we focused on the formation of Kimmelstiel Wilson nodules or diabetic global sclerosis and didn't include the early forms which are essentially same with benign nephrosclerosis, which could be as a consequence of the hypertension or the diabetes mellitus. Also benign glomerulosclerosis has been shown to correlate with age ${ }^{9,23}$. Diabetic glomerulosclerosis has been reported to be the most common cause of end stage renal disease $\mathrm{e}^{18}$. The most common lesion seen in the small vessels was hyaline arteriosclerosis which is found commonly associated with benign sclerosis of the nephrons. Both conditions are commonly described as arterionephrosclerosis. Arterionephrosclerosis refers to the damage of the vessels and kidney parenchymal due to hypertension and aging ${ }^{11}$. Malignant nephrosclerosis is associated with malignant hypertension. It has pathological features that are superimposed on benign arterionephrosclerosis, which includes flea bitten gross appearance, and fibrinoid necrosis of the arteries ${ }^{11}$. The glomeruli might also show thrombi and the arterioles show hyperplastic arteriolosclerosis (Figure 3) which was seen in $11 \%$ of cases in our study.

Renal diseases were the cause of death after autopsies in $10 \%$ of the cases. The main classes of cause of death are cardiovascular diseases and infections. This was similar to the study by Perrone et al. ${ }^{17}$ The major renal diseases in medical certificate of the cause of death were end stage renal disease, obstructive uropathy with chronic kidney disease and chronic glomerulonephritis. Despite the almost ubiquitous renal pathologies at autopsies, they rarely reflect as cause of death or as contributing cause of death as speculated by Perrone et al., who hypothesized that co-morbid renal diseases can actually potentiate cardiovascular deaths ${ }^{17}$. It is known that complications of $\mathrm{CKD}$ and AKI includes cardiovascular diseases, death and $\mathrm{ESRD}^{7}$.

\section{Limitation of study}

The non-availability of electron microscopy and immunofluorescence in reviewing cases for this study means we might have missed out on some important medical renal diseases that are prevalent in our environment. The sample size is relatively small, thus limiting the powers of the conclusions. Despite this limitation we believe this review bring to the fore the histomorphological characteristics of medical renal diseases in kidneys of the deceased.

\section{Conclusion}

The autopsy provides a valuable renal pathology educational tool, as a wide range of medical renal lesions can be seen from kidneys examined at post mortem. More attention should be given to the kidney by the examining pathologist to elucidate the pathological changes which could be as a result of the systemic illness or even contribute to the final cause of death. This will significantly improve data on pattern of renal diseases in our environment.

\section{Financial support and sponsorship}

This study was funded by the authors.

\section{Competing Interests}

Authors declare that they have no competing interests. Authors' Contributions

SAO conceived, designed the study and reviewed H\&Estained slides of the autopsy kidneys, analysed and interpreted the data. MAA designed the study, acquired, analyzed and interpreted the data, reviewed H\&E-stained slides of the autopsy kidneys. JIN designed the study, acquired the data and reported the $\mathrm{H} \& \mathrm{E}$-stained slides. CAO designed and interpreted the data. All authors drafted, revised, read and approved the manuscript.

\section{References}

1. Ijezie Chukwuonye I, Samuel Ogah O, Ndukaife Anyabolu E, Arinze Ohagwu K, Collins Nwabuko O, Onwuchekwa U, et al. Prevalence of chronic kidney disease in Nigeria: Systematic review of populationbased studies. Int J Nephrol Renovasc Dis. 2018;11:165-72.

2. Levey AS, Atkins R, Coresh J, Cohen EP, Collins AJ, Eckardt $\mathrm{KU}$, et al. Chronic kidney disease as a global public health problem: Approaches and initiatives - A position statement from Kidney Disease Improving Global Outcomes. Kidney Int. 2007;72(3):247-59.

3. Bosan I, Ibrahim A, Oguche S, Tuko M, Abdulrasheed M. Characteristics of acute kidney injury in adult patients in a tertiary health facility in northern Nigeria. J Curr Res Sci Med. 2016;2(2):102-8.

4. Esezobor CI, Ladapo TA, Osinaike B, Lesi FEA. Paediatric Acute Kidney Injury in a Tertiary Hospital in Nigeria: Prevalence, Causes and Mortality Rate. PLoS One. 2012;7(12):e51229.

5. Nichols L, Aronica P, Babe C. Are Autopsies Obsolete ? Am J Clin Pathol. 1998;110(2):210-8.

6. Klessens CQF, Woutman TD, Veraar KAM, Zandbergen M, Valk EJJ, Rotmans JI, et al. An autopsy study suggests that diabetic nephropathy is underdiagnosed. Kidney Int. 2010;90(1):149-56.

7. Henriksen KJ. Autopsy kidneys : an overlooked resource. Autops Case Rep. 2018;8(1):8-10.

8. Henriksen KJ. Assessment of kidneys in adult autopsies. Diagnostic Histopathol. 2017;23(3):117-25.

9. Ueda K, Omae T, Hirota Y, Hiyoshi Y, Nakamljra Y, Katsuki S. Epidemiological and clinico-pathological study on renal diseases observed in the autopsy cases in Hisayama population, Kyushu island, Japan. J Chron Dis. 1976;29(3):159-73.

10. Divyashree BN, Venkatesh K, Madhusudhan HR, Hanumantha RB. Pathological spectrum of non-neoplastic diseases in the pathological spectrum of non- neoplastic diseases in the nephrectomy specimens. J Evid based Med Healthc. 2016;1(15):1909-20.

11. Paueksakon P, Fogo AB. Autopsy Renal Pathology. Surg Pathol. 2014;7(3):321-55.

12. Herrera GA, Joseph L, Gu X, Hough A, Barlogie B. Renal Pathologic Spectrum in an Autopsy Series of Patients With Plasma Cell Dyscrasia. Arch Pathol Lab Med. 2004;128(8):875-9.

13. Seisi S El, Gupta R, Clase CM, Forrest DL, Milandinovic M. Renal Pathology at Autopsy in Patients Who Died after Hematopoietic Stem Cell Transplantation. Biol Blood Marrow Transplant. 2003;9(11):6838.

14. Kakkar N, Menon S, Radotra BD. Histomorphology of renal dysplasia — an autopsy study. Fetal Pediatr Pathol. 2006;25(2):73-86.

15. Hailemariam S, Walder M, Burger H-R, Cathomas G, Mihatsch $\mathrm{M}$, Binswanger $\mathrm{U}$, et al. Renal pathology and premortem clinical presentation of Caucasian patients with AIDS : An autopsy study from the era prior to antiretroviral therapy. Swiss Med Wkly. 2001;131(2728):412-7.

16. Kuroda S, Nishida N, Uzu T, Takeji M, Nishimura M, Fujii T, et al. Prevalence of Renal Artery Stenosis in Autopsy Patients With Stroke. Stroke. 2000;31(1):61-5.

17. Perrone ME, Chang A, Henriksen KJ. Medical renal diseases are frequent but often unrecognized in adult autopsies. Mod Pathol. 2017;31(2):365-73.

18. Bonsib SM, Pei Y. The Non-neoplastic Kidney in Tumor Nephrectomy Specimens What Can it Show and What is Important? Adv Anat Pathol. 2010;17(4):235-50. 
19. Gaut JP. Nephrectomy for Non-neoplastic Kidney Diseases. Surg Pathol. 2014;7(3):307-19.

20. Barakat AJ, Drougas JG. Occurrence of congenital abnormalities of kidney and urinary tract in 13,775 autopsies. Urology. 1991;XXXVIII(4):347-50.

21. Henriksen KJ, Meehan SM, Chang A. Non-neoplastic Renal Diseases are Often Unrecognized in Adult Tumor Nephrectomy Specimens A Review of 246 Cases. Am J Surg Pathol. 2007;31(11):1703-8.
22. Truong LD, Shen SS, Park M, Krishnan B. Diagnosing nonneoplastic Lesions in nephrectomy specimens. Arch Pathol Lab Med. 2009;133(2):189-200.

23. Hoy WE, Douglas-Denton RN, Hughson AC, Johnson K, Bertram JF. A stereological study of glomerular number and volume : Preliminary findings in a multiracial study of kidneys at autopsy. Kidney Int. 2003;63(83):S31-7. 